\title{
Molecular Cloning and Characterization of a Novel Gene Specifically Expressed in Gonad
}

\author{
Ming Zhang ${ }^{1}$, Kazuya Matsumoto ${ }^{2,3 *}$, Yumi Yamamoto ${ }^{2}$, \\ Kazuhiro Saeki ${ }^{2,3}$, Yoshihiko Hosoi i, 3, Kehuan Lu' and \\ Akira Iritani, 3 \\ ${ }^{1}$ Animal Reproduction Institute, Guangxi University, Nanning, Guangxi 530005, China \\ ${ }^{2}$ Department of Genetic Engineering, Kinki University, Wakayama 649-6493, Japan \\ ${ }^{3}$ Institute of Advanced Technology, Kinki University, Wakayama 643-0017, Japan
}

\begin{abstract}
We identified a novel gene, termed GSE (gonad-specific expression gene). Nucleotide sequence analysis of GSE CDNA revealed that the open reading frame of 745-bp encodes a protein of 247 amino acids with a predicted molecular mass of $27.6 \mathrm{kDa}$. The deduced amino acid sequence indicated that GSE protein might be a soluble protein in the cytoplasm without a signal peptide. Northern blot analysis showed that this gene was abundantly expressed in mouse testis and slightly expressed in the mouse ovary. RT-PCR analyses indicated that the GSE IRNA in the testis was first detected at Day 14 postpartum, when spermatocytes at mid-pachytene are likely to appear. In situ hybridization confirmed its expression at this stage of spermatogenesis. On the other hand, the GSE mRNA in the ovary was already present at birth, when germ cells are in meiosis. These observations suggest that GSE may be associated with meiosis during gametogenesis.
\end{abstract}

Key words: cDNA, Gametogenesis, Mouse

In mammals, the germ cells develop from primordial diploid cells to haploid gametes through a complex developmental process. The differentiation of germ cells in morphological and biochemical properties is determined by changes of gene expression. It requires a strict program of stage- and cell-specific gene expression in germ cells as well as in surrounding somatic cells. Therefore, it is necessary to study the

Received: July 15,2002

Accepted: August 20, 2002

*To whom correspondence should be addressed.

e-mail:kazum@gene.waka.kindai.ac.jp stage- and cell-specific expression of genes in order to understand the molecular mechanisms of spermatogenesis and oogenesis. Some stage- and cell-specific genes that exert specific rules during gametogenesis have been cloned. A protooncogene ckit, a member of the tyrosine kinase receptor family, is directly involved in the mitotic cell cycle of spermatogonia, which is associated with the survival and proliferation of differentiating type A spermatogonia $[1,2]$. LRTP, a member of the family of leucine-rich repeat proteins, was abundantly expressed in late pachytene and diplotene cells and significantly declined after the first meiotic division, suggesting a functional association of this protein with the prophase of meiosis [3]. Haspin is specifically expressed in haploid round spermatids, and it is believed that haspin plays a role in cell cycle regulation after meiosis in haploid germ cells $[4,5]$. On the other hand, meiosis-specific protein SCP3 is suggested to be linked to inherited aneuploidy in the female germ cell [6]. More recently, two genes, fragilis and Pgc7/stella, were reported to be involved in initiating germ cell competence and specification in proximal epiblast cells by the extra-embryonic ectoderm $[7,8]$.

Recently, the mouse full-length cDNA encyclopedia was reported [9]. This is extremely powerful for analyzing how certain genes cause a given phenotype. In this report, we focus on a novel gonad-specific gene and investigate its expression during gametogenesis in the mouse testis and ovary. 


\section{Materials and Methods}

\section{Cloning of mouse GSE CDNA}

We recently identified a novel gonad-specific partial cDNA (GSE, gonad-specific expression gene) in a previous experiment [10]. This gene showed 100\% similarity with mouse cDNA clone (AK005861 in GenBank ${ }^{\mathrm{TM}}$, 1700011E24 in RIKEN full-length enriched mouse cDNA library). So, we isolated and cloned the full-sequence of this CDNA by RT-PCR from mouse testis. For cDNA cloning of mouse GSE gene, forward primer (5'-GGCACCAATATTGTTTTAT-3') and reverse primer (5'-GGCAAGAGGAGGTCGCAATG-3') were designed on the basis of the sequence of adult testis cDNA clone (AK005861). These primers were used for reverse transcriptase-PCR $\left(94^{\circ} \mathrm{C}\right.$ for 30 second, $55^{\circ} \mathrm{C}$ for 30 second, $72^{\circ} \mathrm{C}$ for 1 minute for 40 cycles) with EX Taq $^{\text {TM }}$ (Takara) and mouse testis mRNA prepared as described in the Quick Prep ${ }^{\mathrm{TM}}$ Micro mRNA Purification Kit (Amersham). The RT-PCR product of the expected size ( 800 base pairs ) was cloned into PGEM-T Easy Vector (Promega) and sequenced. The nucleotide sequence was determined by using a model 310 DNA sequencer (ABI) with $\mathrm{ABI}$ PRSIN BigDye ${ }^{\mathrm{TM}}$ Primer Cycle Sequencing Ready Reaction Kit (ABI). The nucleotide sequence and the deduced amino acid sequence were analyzed and determined through a homology search with GenBank ${ }^{\mathrm{TM}}$, EB, SWISSPROT, and PIR data banks.

\section{Northern blot analysis}

Total RNA was extracted from 9 different mouse tissues by the Trizol / phenol / chloroform extraction method. Poly $(A)^{+}$mRNA was purified by Olgotex (Takara). Two micrograms of mRNA was separated by electrophoresis on $1 \%$ agarose gel containing formaldehyde and transferred onto Hybond- $\mathrm{N}^{+}$nylon membranes (Amersham). The blot was hybridized with random-primed ${ }^{32} \mathrm{P}$-radiolabelled GSE cDNA probe (a 763-bp fragment) according to the manufacturer's instructions of Express-Hybrid ${ }^{\mathrm{TM}}$ buffer (Amersham) and detected using BAS-2500 Bio-Image Analyzer (Fuji Film).

\section{In situ hybridization}

The paraffin-embedded mouse testes were cut into 5$6 \mu \mathrm{m}$ sections and attached to silan-treated slides. The sections were routinely dewaxed and hydrated. After each treatment with $0.3 \%$ Triton- $\mathrm{X}, 0.2 \% \mathrm{HCl}$, and 20 $\mu \mathrm{g} / \mathrm{ml}$ Proteinase $\mathrm{K}$, sections were refixed in $4 \%$ paraformaldehyde, treated with $0.2 \%$ glycine and then $50 \%$ formamide in $2 X$ SSC. GSE sense and antisense RNA probes were synthesized from GSE cDNA (a 763- bp fragment) cloned into pGEM-T Easy Vector with digoxigenin-labeled UTP according to the manufacturer's protocol (Boehringer Mannheim). The hybridization mixture consisted of $50 \%$ deionized formamide, $5 \mathrm{X}$ SSC, $10 \%$ dextran, 1X Denhardt's solution, $100 \mu \mathrm{g} / \mathrm{ml}$ sheared and denatured salmon sperm DNA, $100 \mu \mathrm{g} / \mathrm{ml} \mathrm{E}$. coli tRNA, $10 \mathrm{mM}$ dithiothreitol, and denatured probe. After hybridization overnight at $50^{\circ} \mathrm{C}$, the slides were washed in $4 \mathrm{X} \mathrm{SSC}$ at $42^{\circ} \mathrm{C}$ for 20 minutes and then treated with $20 \mu \mathrm{g} / \mathrm{ml}$ RNase $A\left(37^{\circ} \mathrm{C}, 30\right.$ minutes) to remove the nonhybridized riboprobe. The slides were washed in $2 X$ SSC at $68^{\circ} \mathrm{C}$ for 1 hour and then incubated in $0.2 \mathrm{XSC}$ at $68^{\circ} \mathrm{C}$ for 1 hour. The hybrids were reacted with $1.5 \mathrm{U} / \mathrm{ml}$ anti-digoxigenin Fab fragments conjugated to alkaline phosphatase, and then they were developed with 5bromo-4-chloro-3-indolyl-phosphate-nitroblue tetrazolium (BCIP/NBT) as substrate.

\section{RT-PCR analysis}

Messenger RNA ( $0.5 \mu \mathrm{g}$ each) extracted and purified from mouse testes and ovaries on different postnatal weeks were reverse transcribed using AMV reverse transcriptase (Takara) and random 9-mer primers in a 40 $\mu \mathrm{l}$ reaction mixture. The cDNA from the testis was amplified using specific primers (5'AGCTCCCTCTTGTGCTGAGA-3', 5' TTCAGCTCATAGTCACCTGG-3') with parameters consisting of $94^{\circ} \mathrm{C}$ for 60 seconds, $60^{\circ} \mathrm{C}$ for 60 seconds, and $72^{\circ} \mathrm{C}$ for 60 seconds for 40 cycles. To detect GSE gene expression in the mouse ovary, the nested PCR was performed as follows. First PCR was done with specific primers (5'-GAAGGATGGTGGAGGCTCAC-3', 5'-GGTTGAAGACTGCTTGGGGA-3') under cycling conditions consisting of $94^{\circ} \mathrm{C}$ for 60 seconds, $60^{\circ} \mathrm{C}$ for 60 seconds, and $72^{\circ} \mathrm{C}$ for 60 seconds for 40 cycles. One microliter of reaction mixture of the first PCR products was put into tubes with a reaction containing the inner set of primers (5'-AGCTCCCTCTTGTGCTGAGA-3', 5'TTCAGCTCATAGTCACCTGG-3'). Cycling conditions were 40 cycles of $94^{\circ} \mathrm{C}$ for 60 seconds, $60^{\circ} \mathrm{C}$ for 60 seconds, and $72^{\circ} \mathrm{C}$ for 60 seconds. All amplified products were separated on a $2 \%$ agarose gel by electrophoresis. RT-PCR for mouse G3PDH (glyceraldehyde 3-phosphate dehydrogenase) gene was performed using Clontech primers. Three independent experiments were done in this study.

\section{Southern blot analysis of GSE gene}

Ten micrograms of genomic DNA from mouse testes was digested with either BamHI, EcoRl, HindIII, Sacl, or 


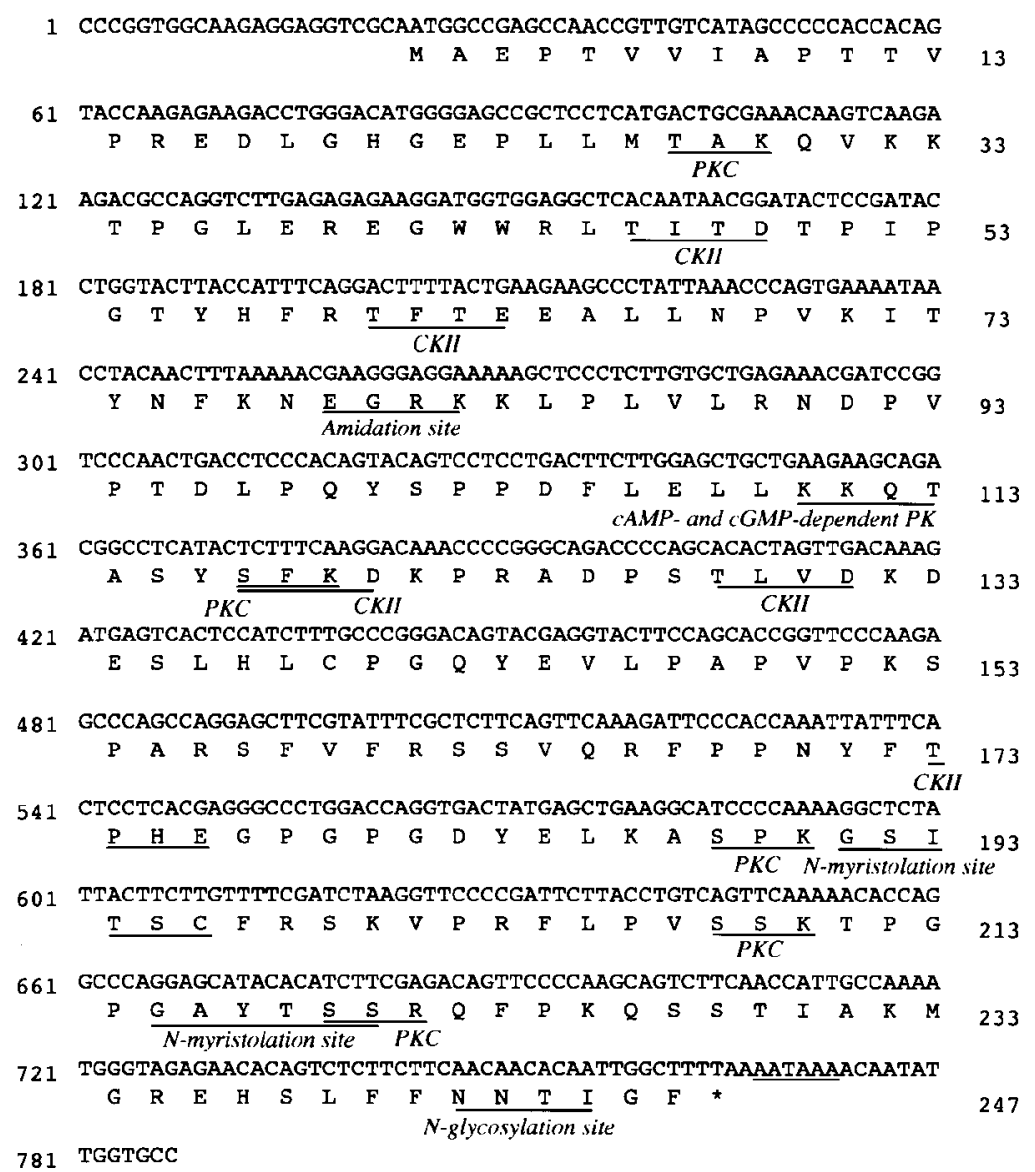

Fig. 1. Nucleotides and deduced amino acids of mouse GSE. The nucleotides and deduced amino acids are numbered on the left and right, respectively. The putative polyadenylation sequence is underlined. The stop codon is marked with an asterisk. cAMP- and cGMP-dependent PK: cAMP- and cGMP-dependent protein kinase phosphorylation site. PKC: protein kinase C phosphorylation site. CKII: casein kinase II phosphorylation site.

$X$ bal, separated on $1 \%$ agarose gel by electrophoresis and transferred to a nylon filter (Hybond $\mathrm{N}^{+}$, Amersham). Hybridization and detection were performed with $\mathrm{ECL}$ gene detection procedures (Amersham) according to the manufacturer's recommended procedure.

\section{Results}

Molecular cloning and structural features of mouse GSE

The nucleotide sequences of mouse GSE cDNA and the deduced amino acid sequences are shown in Fig. 1. The open reading frame of mouse GSE extends 745-bp and encodes a protein of 247 amino acids residues with a predicted molecular mass of $27.6 \mathrm{kDa}$. The $\mathrm{N}$ terminal region of amino acid sequences of mouse GSE showed no similarity to known proteins on the data base, whereas the C-terminal region (amino acids 107235) of GSE showed similarity with possible alpha collagen-related protein (accession number Q9NE64). Computer-assisted sequence analysis showed that GSE contained one potential glycosylation and several protein kinase C, CAMP- and CGMP-dependent, and casein kinase-II phosphorylation sites. It is also highly basic ( $\mathrm{pl}=9.84$; the content of basic residues is $27.3 \%$ ) with no signal peptide, indicating that it might be a soluble protein in the cytoplasm.

\section{Gonad-specific expression of mouse GSE}

The tissue distribution of GSE gene expression was examined by Northern blot analysis of poly $(A)^{+}$RNAs 


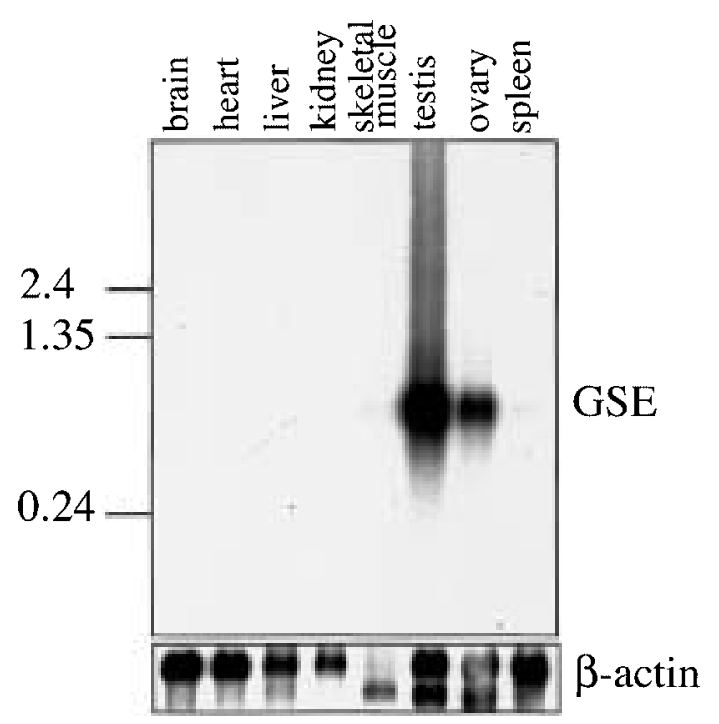

Fig. 2. Expression of GSE mRNA in mouse tissues. Two micrograms of Poly $(\mathrm{A})^{+}$RNA from various tissues of adult mice were subjected to Northern blot analysis using the mouse GSE cDNA probe.

from various tissues of adult mice using the full-length of GSE cDNA as a probe. One transcript of about 0.8-kbp was exclusively detected in the testis as shown in Fig. 2. Furthermore, there was GSE mRNA in the mouse ovary that showed a very weak signal with this probe (Fig. 2). No transcript was observed in somatic tissues such as the heart, liver, kidney, lung, brain, skeletal muscle, and spleen. The blots were stripped and reprobed with $\beta$ actin cDNA to ensure equivalent mRNA loading.

\section{Expression pattern of mouse GSE during testis and ovary development}

RT-PCR analysis of mRNA from 0-day-old to adult mouse testes (49-day-old) was performed to confirm the developmental change of GSE mRNA expression in the mouse testis and ovary. As shown in Fig. 3, GSE mRNA in the mouse testis was expressed initially at Day 14 after birth and also expressed thereafter, corresponding to the appearance of spermatocytes at mid-pachytene. On the other hand, a substantial increase of GSE gene expression in the ovary was observed from newborn, prepubertal, and sexually mature mice.

\section{Stage-specific distribution of mouse GSE}

To investigate further whether the GSE gene is specifically expressed in germ cells and the localization of GSE mRNA in the testis, we examined the GSE

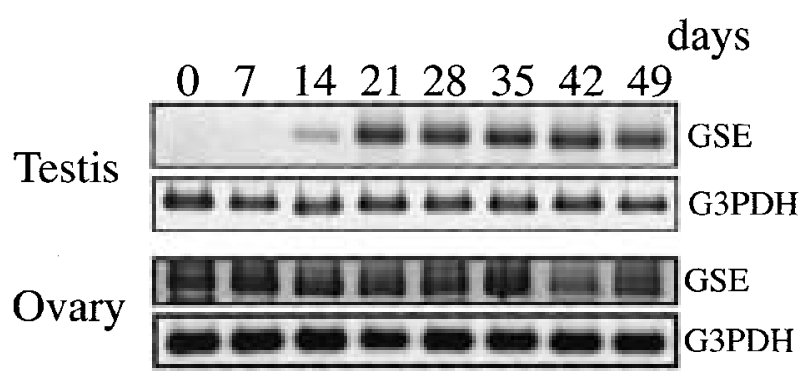

Fig. 3. Developmental changes of GSE mRNA expression in mouse testes and ovaries. mRNA $(0.5 \mu \mathrm{g}$ each) from mouse testes and ovaries on different postnatal weeks (W) were analyzed by RT-PCR as described under "Material and Methods".

mRNA localization in the mouse testis by in situ hybridization using mouse GSE cDNA as a probe. The GSE mRNA was distributed within the cytoplasm of spermatocytes I (pachytene), round spermatids, and elongated spermatids, either cell of which was observed in stage VI-VII or VII-VIII tubules (Fig. 4B). No significant signal was detected in hybridization using a sense probe (Fig. 4A).

\section{Genomic southern blot analysis of mouse GSE}

To determine if the mouse genome contains more than one GSE gene, we did Southern blot analysis. The sizes of the resulting fragments corresponded to the restriction fragments of the database sequence (AC084383), which includes GSE genomic DNA (Fig. 5). These results indicate that the GSE gene is a single copy in the mouse genome and not an intronless gene like the haspin gene $[4,5]$. The latter result is consistent with the preliminary result indicated by genomic PCR with specific primers for GSE cDNA (data not shown).

\section{Discussion}

We report here the cloning and characterization of a novel mouse cDNA that is expressed in the testis and ovary but is not expressed in the somatic tissues in mice. It is therefore referred to here as GSE (gonad-specific expression gene). This GSE gene has one homology region; the $\mathrm{C}$-terminal region displays homology to the possible alpha collagen-related protein. Furthermore, GSE contains many potential target sites for protein kinases, i. e. CAMP- and cGMP-dependent protein kinase phosphorylation sites, protein kinase $C$ phosphorylation site, and casein kinase-II phosphorylation site. These suggest that GSE might have biological activity in 


\section{Sense riboprobe}

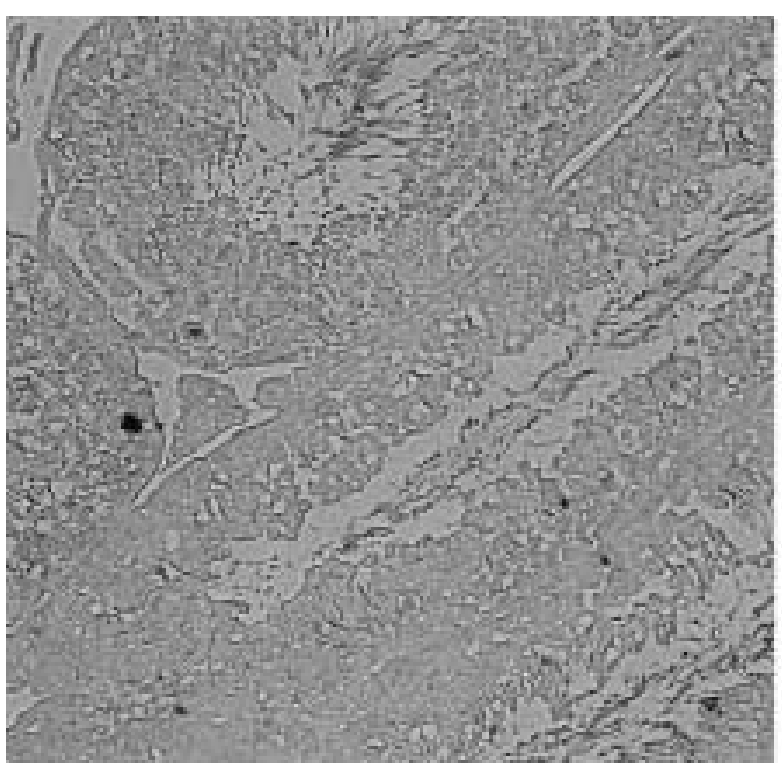

\section{Antisense riboprobe}

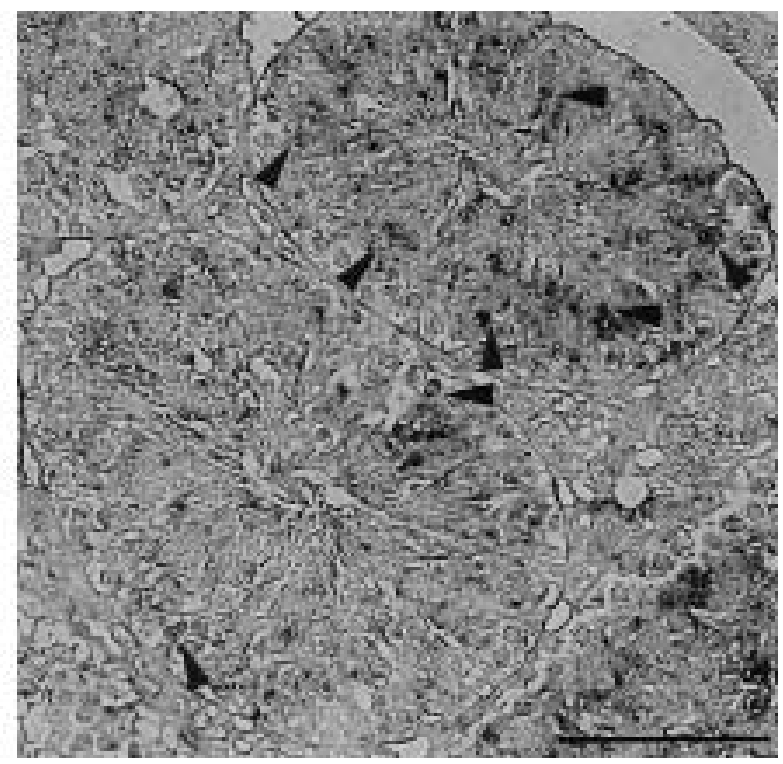

Fig. 4. The distribution of GSE mRNA in mouse testis. A riboprobe was transcribed from mouse full (787-bp) GSE cDNA and used for in situ hybridization. Signals are visible in the cytoplasm of spermatocytes I (pachytene), round spermatids, and elongated spermatids (arrowheads). Scale bar is $100 \mu \mathrm{m}$.

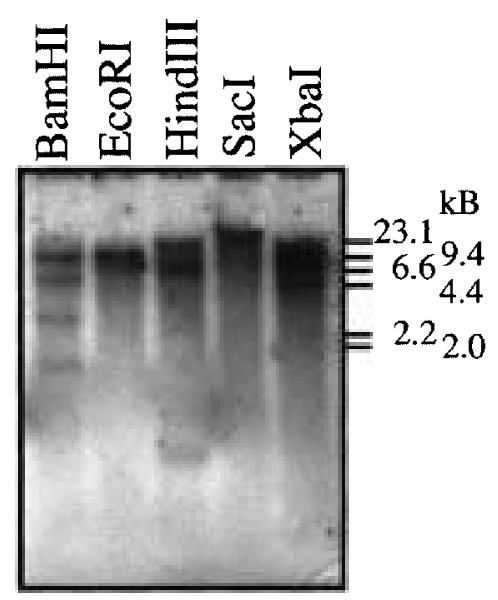

Fig. 5. Southern blot analysis of GSE gene. Mouse genomic DNA $(10 \mu \mathrm{g})$ was digested with either BamHI, EcoRI, HindIII, SacI, or XbaI, fractionated on a $1 \%$ agarose gel, then transferred to a nylon membrane. The membrane was probed with mouse GSE cDNA probe.

phosphorylated or dephosphorylated form. Protein phosphatases are involved in the regulation of cell proliferation and differentiation by co-operation with protein kinases [11]. Protein phosphorylation seems to be involved as an important molecular mechanism in the regulation of spermatogenesis because some studies showed that several protein kinases and phosphatases are expressed specifically in the testis. Recently, Varmnza S. et al. (1999) reported that target disruption of the protein phosphatase PPLR gene, which encodes an isoform of type- 1 serine protein phosphatase, induces meiotic defects in the testis [12]. This finding suggests that the protein phosphatase is involved in the stagespecific regulation of spermatogenesis. Further study to determine the GSE protein function in gametogenesis will be necessary.

Northern blot analysis revealed that the mouse GSE gene is expressed exclusively in both the testis and ovary. Although only a faint band of mRNA was detectable in the ovary, GSE gene expression in the testis was much higher than its expression in the ovary. Thus, it is possible that GSE has physiologically important role in the testis. Spermatogenesis is a complex differential process which involves changes in gene expression. Generally, in order to satisfy the developmental demands of the tissue, the differentiation of male gametes requires the expression of many germ cell-specific proteins at specific stages of development [13-15]. The expression patterns of many genes that are expressed in the testis are cell type-specific or 
stage-specific $[16,17]$. In our present study, we demonstrated the developmentally regulated pattern of expression of the GSE mRNA in the mouse testis, which exemplifies such a pattern. In the mouse seminiferous tubule, spermatogenetic cells of the same development stage form a concentric layer of cells. Mitotic proliferation of stem cells, which are seen in the initial phase of spermatogenesis, occurs in the basal compartment. Successively, meiosis and spermiogenesis occur toward the inside of the seminiferous tubules. Therefore, it is generally possible to specify the developmental stage of germ cells on the basis of their sizes, shapes, and spatial distribution [18]. Our in situ hybridization analysis in the mouse testis demonstrated that the GSE mRNA expresses in spermatocytes I (pachytene), round spermatids, and elongated spermatids. RT-PCR analysis of testes from 0 to 49-day-old mice indicated that the GSE mRNA became detectable around 14 days after birth, which almost paralleled with the stage of spermatogenesis, when mouse spermatocytes undergo meiosis and differentiation into haploid round spermatids. Therefore, the characteristic distribution and developmental expression of mouse GSE gene indicates that the regulation of GSE is involved in the meiotic division.

Although the expression of the GSE gene was detected in the adult mouse ovary, only a low level of GSE mRNA was seen. RT-PCR analysis of ovaries from 0 to 49-day-old mice indicated that the GSE mRNA was already detectable just after birth, when germ cells are in meiosis. However, further investigation will be needed to confirm if the GSE gene can regulate meiosis of mammalian female germ cells.

In summary, GSE is a novel gene that is specifically expressed in the mouse gonad, and abundantly expressed in the testis, suggesting an important role for the GSE gene in meiosis of mammalian germ cells.

\section{Acknowledgment}

Part of this work was supported by a fellowship grant from the Japan Society for the promotion of Science (JSPS, L-01556).

\section{References}

1) Manova, K., Nocka, K., Besmer, P. and Bachvarova, R.E. (1990): Gonadal expression of c-kit encoded at the W locus of the mouse. Development, 110, 1057-1069.

2) Yoshinaga, K., Nishikawa, S., Ogawa, M., Hayashi, S., Kunisada, T., Fujimoto, T. and Nishikawa S. (1991): Role of c-kit in mouse spermatogenesis: identification of spermatogonia as a specific site of c-kit expression and function. Development, 113, 689-699.

3) Xue, J.C. and Goldberg, E. (2000): Identification of a novel testis-specific leucine-rich protein in humans and mice. Biol. Reprod., 62, 1278-1284.

4) Tanaka, H., Yoshimura, Y., Nozaki, M., Yomogida, K., Tsuchida, J., Tosaka, Y., Habu, T., Nakanishi, T., Okada, M., Nojima, H. and Nishimune, Y. (1999): Identification and characterization of a haploid germ cell-specific nuclear protein kinasa (haspin) in spermatid nuclei and its effects on somatic cells. J. Biol. Chem., 274, 17049-17057.

5) Tanaka, H., Iguchi, N., Nakamura, Y., Kohroki, J., Egydio, C. and Nishimune, Y. (2001): Cloning and characterization of human haspin gene encoding haploid germ cell-specific nuclear protein kinase. Mol. Hum. Reprod., 3, 211-218.

6) Yuan, L., Liu, J.G., Hoja, M.R., Wilbertz, J., Nordqvist, K. and Hoog, C. (2002): Female germ cell aneuploidy and embryo death in mice lacking the meiosis-specific protein SCP3. Science, 296, 1115-1118.

7) Saitou, M., Barton, S.C. and Surani, M.A. (2002): A molecular programme for the specification of germ cell fate in mice. Nature, 418, 293-300.

8) Sato, M., Kimura, T., Kurokawa, K., Fujita, Y., Abe, K., Masuhara, M., Yasunaga, T., Ryo, A., Yamamoto, M. and Nakano, T. (2002): Identification of PGC7, a new gene expressed specifically in preimplantation embryos and germ cells. Mech. Dev., 113, 91-94.

9) The RIKEN Genome Exploration Research Group Phase II Team and the FANTOM Consortium. (2001): Functional annotation of a full-length mouse cDNA collection. Nature, 409, 685-690.

10) Matsumoto, K., Nakagami, K., Otake, S., Tanaka, H., Hashimoto, Y., Yamada, S., Saeki, K., Hosoi, Y. and Iritani, A. (2001): Identification of genes expressed in mouse unfertilized and fertilized eggs using Differential Display of RNA. J. Fertil. Implant., 18, 5-7.

11) Hunter, T. (1995): When is a lipid kinase not a lipid kinase? When it is a protein kinase. Cell, 83, 1-4.

12) Varmuza, S., Jurisicova, A., Okano, K., Hudson, J., Boekelheide, K. and Shipp, E.B. (1999): Spermiogenesis is impaired in mice bearing a targeted mutation in the protein phosphatase 1cgamma gene. Dev. Biol., 205, 98-110.

13) Millette, C.F. and Moulding, C.T. (1981): Cell surface marker proteins during mouse spermatogenesis, twodimensional electrophoretic analysis. J. Cell Sci., 48, 367382.

14) Hecht, N.B. (1998): Molecular mechanisms of male germ cell differentiation. Bioessays, 20, 555-561.

15) Eddy, E.M. and O'Brien, D.A. (1997): Gene expression during mammalian meiosis. In: Current Topics in Developmental Biology 37 (Handel, M.A. ed.), pp 141200, Academy Press, San Diego.

16) Willison, K. and Ashworth, A. (1987): Mammalian spermatogenetic gene expression. Trend. Genet., 3, 351355.

17) Wolgemuth, D.J. and Watrin, F. (1991): List of cloned 
mouse genes with unique expression patterns during spermatogenesis. Mamm. Genome, 1, 283-288.

18) Ohsugi, M., Kuramochi, S., Matsuda, S. and Yamamoto, T. (1997): Molecular cloning and characterization of a novel cytoplasmic protein-tyrosine phosphatase that is specifically expressed in spermatocytes. J. Biol. Chem., 52, 33092-33099. 